\title{
An agent based architecture for high-risk neonate management at neonatal intensive care unit
}

Jaleh Shoshtarian Malak ${ }^{1}$, Reza Safdari ${ }^{2}$, Hojjat Zeraati $^{3}$, Fatemeh Sadat Nayeri ${ }^{4}$, Niloofar Mohammadzadeh ${ }^{5}$, Seide Sedighe Seied Farajollah ${ }^{6}$

${ }^{1}$ Ph.D. Candidate of Medical Informatics, Department of Medical Information Management, Faculty of Allied Sciences, Tehran University of Medical Science, Tehran, Iran

${ }^{2} \mathrm{Ph}$.D. of Health Information Management, Professor, Department of Medical Information Management, Faculty of Allied Sciences, Tehran University of Medical Science, Tehran, Iran

${ }^{3}$ Ph.D. of Biostatistics, Professor, Department of Epidemiology and Biostatistics, School of Public Health, Tehran University of Medical Science, Tehran, Iran

${ }^{4}$ Ph.D. of Neonatology, Professor, Maternal-Fetal \& Neonatal Research Center, Tehran University of Medical Science, Tehran, Iran

${ }^{5} \mathrm{Ph} . \mathrm{D}$. of Health Information Management, Assistant Professor, Department of Medical Information Management, Faculty of Allied Sciences, Tehran University of Medical Science, Tehran, Iran

${ }^{6} \mathrm{Ph}$.D. Candidate of Health Information Management, Department of Medical Information Management, Faculty of Allied Sciences, Tehran University of Medical Science, Tehran, Iran

Type of article: Original

\begin{abstract}
Background: In recent years, the use of new tools and technologies has decreased the neonatal mortality rate. Despite the positive effect of using these technologies, the decisions are complex and uncertain in critical conditions when the neonate is preterm or has a low birth weight or malformations. There is a need to automate the high-risk neonate management process by creating real-time and more precise decision support tools.

Objective: To create a collaborative and real-time environment to manage neonates with critical conditions at the NICU (Neonatal Intensive Care Unit) and to overcome high-risk neonate management weaknesses by applying a multi agent based analysis and design methodology as a new solution for NICU management.

Methods: This study was a basic research for medical informatics method development that was carried out in 2017. The requirement analysis was done by reviewing articles on NICU Decision Support Systems. PubMed, Science Direct, and IEEE databases were searched. Only English articles published after 1990 were included; also, a needs assessment was done by reviewing the extracted features and current processes at the NICU environment where the research was conducted. We analyzed the requirements and identified the main system roles (agents) and interactions by a comparative study of existing NICU decision support systems. The Universal Multi Agent Platform (UMAP) was applied to implement a prototype of our multi agent based high-risk neonate management architecture.

Results: Local environment agents interacted inside a container and each container interacted with external resources, including other NICU systems and consultation centers. In the NICU container, the main identified agents were reception, monitoring, NICU registry, and outcome prediction, which interacted with human agents including nurses and physicians.

Conclusion: Managing patients at the NICU units requires online data collection, real-time collaboration, and management of many components. Multi agent systems are applied as a well-known solution for management, coordination, modeling, and control of NICU processes. We are currently working on an outcome prediction
\end{abstract}

\section{Corresponding author:}

Professor Dr. Reza Safdari, Ghods Ave, Farredanesh Alley, No \#17, Department of Medical Information Management, Faculty of Allied Sciences, Tehran University of Medical Sciences, Teheran, Iran.

Tel: +98.2188983025, Email: rsafdari@tums.ac.ir

Received: August 28, 2017, Accepted: December 14, 2017, Published: January 2018

iThenticate screening: December 08, 2017, English editing: December 17, 2017, Quality control: December 18, 2017 This article has been reviewed / commented by four experts

(C) 2018 The Authors. This is an open access article under the terms of the Creative Commons Attribution-NonCommercialNoDerivs License, which permits use and distribution in any medium, provided the original work is properly cited, the use is non-commercial and no modifications or adaptations are made. 
module using artificial intelligence techniques for neonatal mortality risk prediction. The full implementation of the proposed architecture and evaluation is considered the future work.

Keywords: Neonatal Intensive Care, Software Design, Neonatal Mortality, Clinical Decision Support Systems, Health Information Technology

\section{Introduction}

High-risk neonates are treated in the Neonatal Intensive Care Unit (NICU) according to their condition. Decisionmaking about preterm neonates with high mortality probability is often very expensive and has many social consequences on the patient's family (1). The suitable treatment level is chosen by physicians based on the patient's existing medical findings and their past medical research knowledge $(2,3)$. In complex conditions when the neonate is preterm, has a low weight, or has malformations, the decisions are uncertain and it is difficult to choose a moderate or aggressive treatment $(4,5)$. Monitoring patients at the intensive care unit requires online data collection and real-time collaboration and management of many components at the NICU. In this section, data should be delivered fast and decisions should be made accurately (6). Multi agent systems are composed of interacting intelligent software components with the main goal of solving a common and complex problem $(7,8)$. The features of multi agent systems such as being autonomous, proactive, and collaborative and their negotiation capabilities make them good choices for managing real time, complex, and critical environments like hospital intensive care units $(6,9,10)$. In this paper, we introduced a multi agent based high-risk neonate management architecture with regard to multi agent system advantages. We also deployed a prototype application using the UMAP (Universal Multi Agent Platform). Multi-agent based intensive care management systems can be divided into two groups: a) clinical management systems and b) research systems. Multi agent based clinical management systems are used for prognosis, diagnosis, and treatment while research based systems are used to extract medical facts, relations, patterns, and trends from medical data $(11,12)$. Multi agent systems are a great solution to real-time distributed systems; they can be used to detect abnormal neonatal conditions and provide appropriate alarms $(13,14)$. FIPA is an IEEE computer society standard that develops standards for multi agent systems and their interoperability. The main goal of the standard is to provide specifications on how multi agent platforms should be implemented, and how agents should execute and communicate. FIPA agents rely on three main roles: Agent Management System (AMS) that enables registering and tracking of agents and provides supervisory control over access for utilizing the platform. The AMS utilizes an agent called Directory Facilitator (DF) as a directory of agents and services; agents use DF to register themselves and to find other agents and services for interaction. FIPA also provides an agent communication channel for basic contact between agents inside and outside the platform $(9,15,16)$. GAIA is a methodology for agent-oriented analysis and design. GAIA is an established and general approach that can be applied to a wide range of applications. The main steps in GAIA methodology include requirement analysis, role analysis, interaction analysis, and design $(15,17)$. The UMAP is a FIPA compliant multi agent based framework providing interfaces for multi agent systems implementation. UMAP can be extended to implement custom agent based solutions and is based on .Net Framework $(18,19)$. In this research, we used the UMAP platform to implement our multi agent based high-risk neonate management architecture. A conceptual architecture for neonatal knowledge management and outcome prediction was presented in (3) to support ethical treatment decisions based on patient information, physicians knowledge and parents priorities at NICU. In (2), parents interact with the system, and their priorities are identified and used for neonatal treatment recommendations. The outcome predication module is implemented using evidence based artificial intelligence techniques. The correlation between the indictors and outcomes is analyzed and used for prediction of neonatal death and length of stay. The patient's status and the physician's and parents' opinion are stored in the knowledge base. Viewing treatments made by the physicians, and their next plans by parents will help them make critical decisions for their neonate (2). The work which was done in (2) was extended in (20) by using new methodologies and adding new artificial intelligence algorithms for neonate outcome prediction in the NICU. With multi agent systems, we can transform the passive behavior of system components into an autonomous and proactive environment, which will help us to create a high quality fast response system (6). In (21), by extending (6), a decision support system is presented for the NICU that specifies the neonate's nutrition plan, mortality probability, and height and weight percentiles, and is also used for treatment of neonatal jaundice. The use of multi agent systems and the base platform has been introduced in (6) and a system for automating data gathering, and automatic data processing and transforming is proposed. An intelligent multi agent based system is presented in (13) which analyses the neonate's physiological data and new data trends are detected by the system. Communication between agents is done by passing requests between service consumers and service providers and the related communication is then validated. Access to different data bases is managed using an agent server that is responsible for data gathering and transformation. In this study, we reviewed the relevant papers and proposed an architecture in the field of neonatal intensive care decision support systems. We also made a 
comparative analysis to introduce a high-risk neonate management architecture based on multi agent systems. A prototype for a physician and parent decision support system was proposed with the ability to view the neonate's latest condition, predict high-risk neonates, and determine the prognosis of high-risk neonates. The designed system specification and requirement analysis is based on a comparative review of existing neonatal decision support systems and neonatal management processes in the Maternal Fetal and Neonatal Research Center of Tehran University of Medical Sciences where this study was conducted. The proposed architecture is abstract and could be applied to other healthcare scenarios. The main objective of this study was to overcome high-risk neonate management weaknesses observed in the critical, real-time and collaborative environment of the NICU. Considering these weaknesses and in order to provide a real-time solution for monitoring and collaboration of the components in the NICU, we applied a multi agent based analysis and design methodology as a new solution for NICU management.

\section{Material and Methods}

This study was a basic research for medical informatics method development that was carried out in 2017 . The analysis and design of the proposed architecture was based on the GAIA methodology, a well-known agent based modeling methodology. The GAIA methodology models and their relationship are shown in Figure 1. This methodology consists of two main stages: the analysis phase and the design phase. In the analysis phase, the first stage is requirement statement. Requirement analysis was done by reviewing articles on NICU Decision Support Systems. PubMed, Science Direct and IEEE databases were searched. Only English articles published after 1990 were included. The overall search strategy was to retrieve articles that included terms related to "NICU Decision Support System" or "Neonatal Decision Support System" in their title or abstract. Fifty-seven articles were found in the initial database search, of which 29 were excluded after title review and 7 were excluded after their abstracts were assessed by professionals. The search was completed by adding 5 articles relevant to our study to enrich the discussion, resulting in 26 selected articles. The selected articles were reviewed and the system's main process, features, and requirements were extracted. Also, a needs assessment was done by reviewing the extracted features and the current process at the NICU environment where the research was conducted. All the identified requirements were listed for role model and interaction model analysis. The main system roles and interactions were identified by requirement analysis and an agent model was presented for high-risk neonate management. After identifying the main agents, their functionality and their interactions, the high-risk neonate management architecture was presented. A prototype application was implemented using the UMAP framework which provides features for agent management and messaging. The presented system features were compared with other existing NICU decision support systems, identified at the review stage considering their main functionalities and features at the discussion section. The overall strategy for this research is shown in Figure 2.

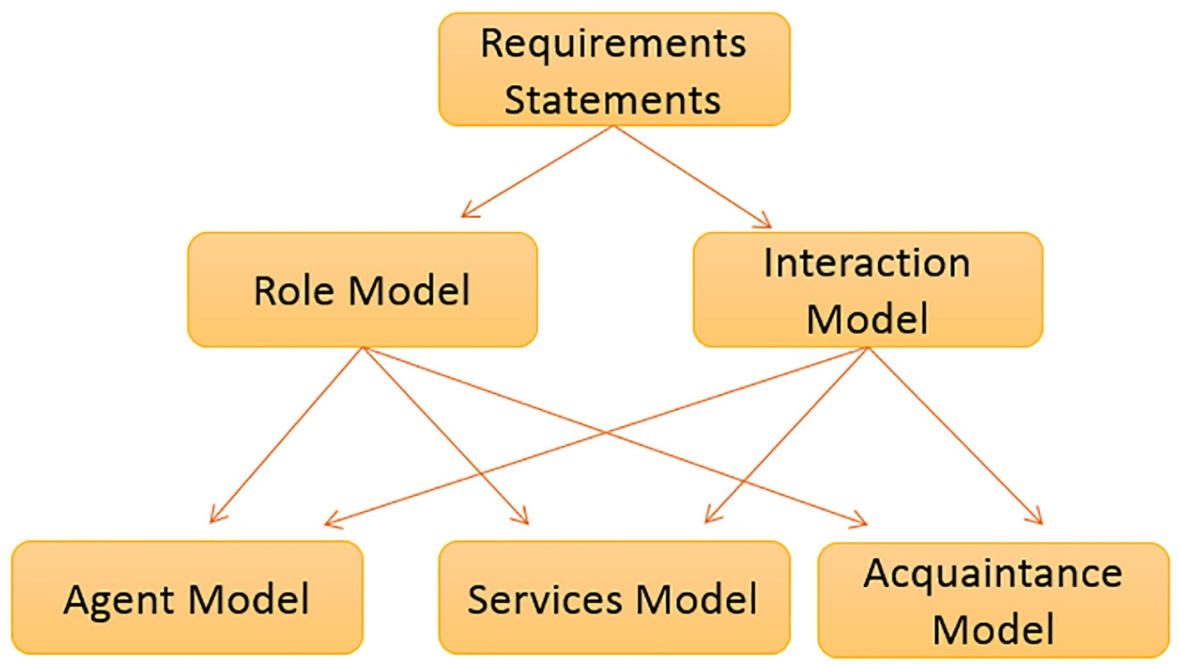

Figure 1. GAIA Methodology Models Relationship (22) 


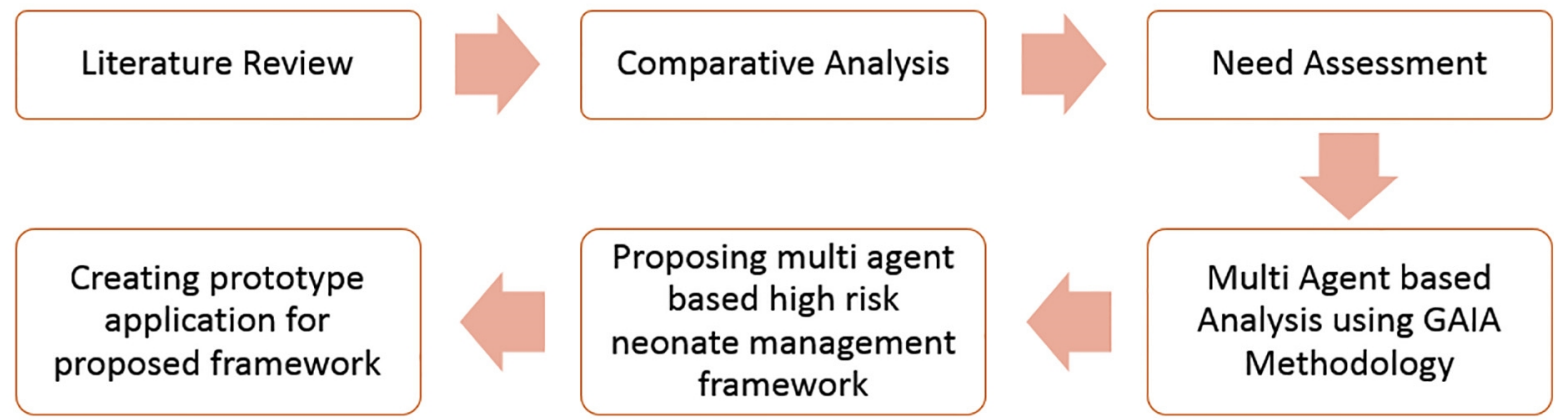

Figure 2. Research strategy for agent based high-risk neonate management architecture

\section{Results}

\subsection{The multi agent based high-risk neonate management architecture}

A framework was presented for managing high-risk neonates considering the extracted roles in the needs assessment. The architecture was based on the UMAP multi agent platform. NICU local environment agents interacted inside a container and each container interacted with external resources, including other NICU systems and consultation centers. In the NICU container, the main identified agents were reception, monitoring, NICU registry, and outcome prediction, which interacted with human agents including nurses and physicians. Laboratory, radiography and medical devices were connected to the NICU registry through some middleware warped as agents. Agent Management System (AMS), Directory Facilitator (DF) and Message Transport System (MTS) were FIPA compliant agents added to the model in order to manage, register, and enable messaging of the agents. Figure 3 shows the multi agent based high-risk neonate management architecture. The system's main users are the healthcare providers including nurses and physicians. They can use the system for data entry, monitoring patients, and reporting and also as a dashboard for patient status prediction. The functionality of the main agents operating in the proposed architecture are described in the following:

1) Reception Agent - retrieves/registers the neonate's mother and demographic data, and if the patient has an electronic health record, it will be retrieved or registered as a new medical record in the Neonatal Registry. The neonate's and the mother's data are sent to the Neonatal Registry after confirmation, or they will be retrieved in the Reception Agent in case they existed from the previous referrals.

2) Neonatal Registry is a neonatal registry system wrapped as an agent in such a way that the reception agent stores/retrieves patient data. Moreover, the data of the radiography, laboratory tests, and medical devices are stored in the neonatal registry. The neonatal registry is connected to a data repository for storing these data and the repository is further connected to a data warehouse system for reporting and calculating outcome measures.

3) Monitoring Agent - collects the patient's reception data and radiography and laboratory test results and medical device data from neonatal registry and sends alarms if the data items are out of the normal range. Health care providers can view the patient's latest status through this agent.

4) Outcome Prediction Agent - collects the latest neonatal data and measurements from neonatal data warehouse, predicts neonate's outcomes, and specifies high risk neonates using artificial intelligence techniques.

5) Data Repository and Data warehouse: Data repository consists of a database which stores neonatal demographic and admission data and daily neonatal transaction. Then, the transactional data is used to build a data warehouse for storing summarized data for data analysis.

6) Agent Management System (AMS) is a FIPA compliant UAMP component. It is responsible for loading agents, and provides a runtime environment for agent operation inside a container. Message Transport Service and Directory Facilitator run under the control of AMS.

7) Directory Facilitator (DF): All the agents within the local container register their information in the DF, and it also holds the agent information of other containers which interact with this container. This agent initiates upon container creation.

8) Message Transport Service (MTS) is a FIPA compliant agent responsible for messaging between agents in a container and remote container agents. This agent enables sending messages and listening for incoming messages and is also responsible for serialization/deserialization of XML messages.

The overall sequence of the system process is as follows: when a neonate is admitted to the NICU, the patient's demographic and past medical history will be retrieved and updated from the neonatal registry. If no previous record 
exists, the new data will be sent to the neonatal registry. Physicians and nurses register data in the neonatal registry while visiting patients. The results of laboratory tests and the data obtained from vital signs devices are also registered in the neonatal registry. The monitoring agent checks the neonate's data at time intervals, and if out of norm data appear, it will trigger an alarm. Physicians and nurses can monitor the patient's status using the monitoring agent. The patient's data are stored in a data repository and then a data warehouse is built over the data repository. The outcome prediction agent uses the stored and analyzed data in order to predict high-risk neonates.

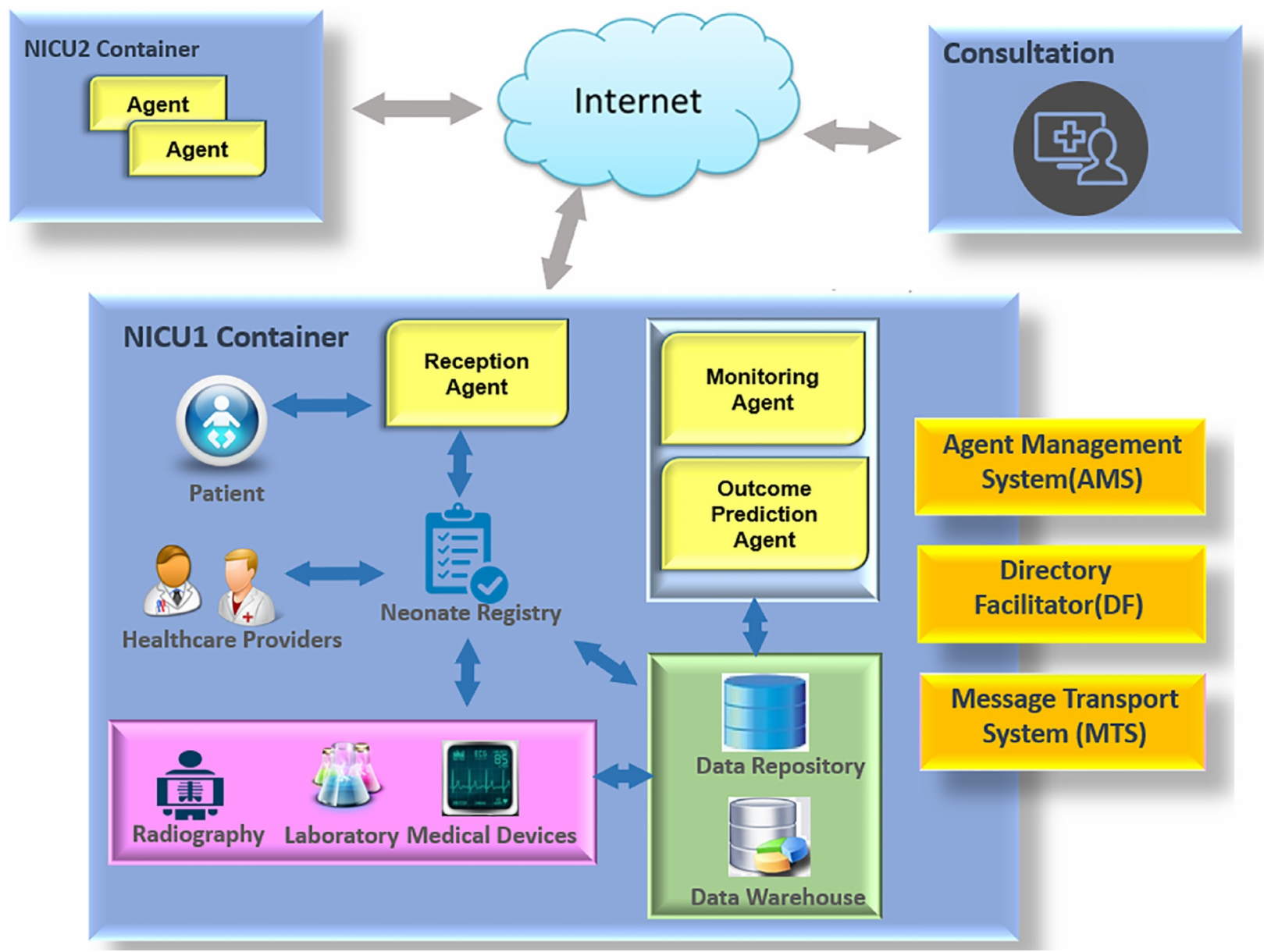

Figure 3. Multi agent based high risk neonate management architecture

\subsection{A prototype system for high risk neonate management}

A prototype high-risk neonate management system based on multi-agent systems was implemented using the UMAP framework. Figure 4 shows the class diagram of the NICU container for management of high-risk neonates. The system's main agents in the NICU container are the patient, reception, healthcare provider, monitoring, and outcome prediction. These agents inherit the abstract UMAP agent class and implement Run and Handle Message methods. The Agent Management System, Directory Facilitator, and Message Transport System are used as FIPA compliant modules for managing, registering, and messaging of the agents. The prototype application is created using the .Net Framework development environment. 

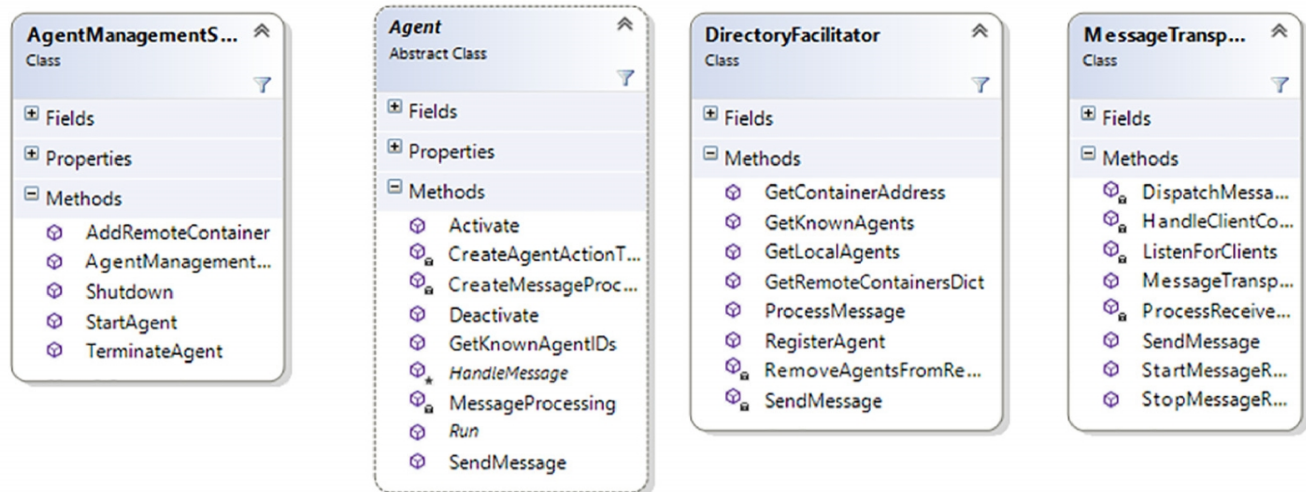

$\Delta$

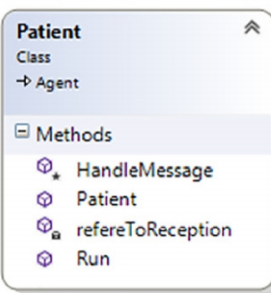

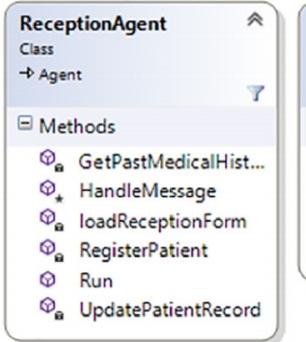

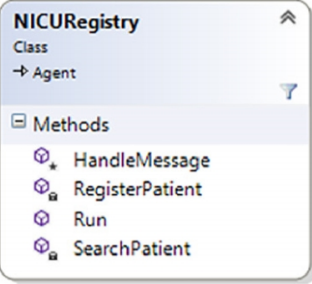

$\Phi_{\mathrm{B}}$ SearchPatient

Figure 4. Class Diagram for a high-risk neonate management NICU container

\section{Discussion}

Decisions about neonates' treatment plans at neonatal intensive care units are complex and uncertain in critical conditions when the neonate is preterm or has a low birth weight or malformations. There is a need to automate the high-risk neonate management process by creating real-time and more precise decision support tools. In this research, we proposed a multi agent based high-risk neonate management framework by applying multi-agent system modeling and control. The main identified agents were reception, monitoring, NICU registry, and outcome prediction, which interacted with human agents including nurses and physicians. Laboratory, radiography and medical devices were connected to the NICU registry through some middleware warped as agents. Agent Management System (AMS), Directory Facilitator (DF) and Message Transport System (MTS) are FIPA compliant agents added to the model for management, registration, the messaging of agents. We deployed an application prototype using the UMAP platform. Unlike other agent development tools like JADE and SPADE, this prototype was based on the .Net Framework development environment, because it is easier to develop and maintain and is less costly. Table 1 compares NICU decision support system architectures based on functional features including knowledge management, alarming, artificial intelligence techniques, multi agent systems, service oriented architecture, data warehouse, outcome prediction, parent decision support, physician decision support, and monitoring and treatment recommendation. Hybrid approaches have been used in patient monitoring, risk detection, and intensive care. Using these approaches could reduce the process time, caregiver's expertise cost and medication errors $(23,24)$. Combining decision support systems, data mining techniques, and multi agent systems provide promising solutions for decision making. Recently, multi agent systems combined with decision support systems and knowledge management techniques have been widely used in intensive care systems, including hospitalacquired infection surveillance in the ICU (25), modeling ICU processes using Intuitionistic Fuzzy Expert Decision Support System (23), remote health care monitoring for risk detection and decision making (24), and dynamic aggregation of medical research data from distributed sources applied in neonatal intensive care (26). The proposed architecture in this study is also considered a multi-disciplinary approach to support decision making and process management in the neonatal intensive care complex environment. 
Table 1. Comparison of neonatal intensive care architecture based on functional features

\begin{tabular}{|l|l|l|l|l|l|l|l|l|l|l|l|l|}
\hline $\begin{array}{l}\text { Ref. } \\
\text { no. }\end{array}$ & $\begin{array}{l}\text { Knowledge } \\
\text { management }\end{array}$ & Alarming & $\begin{array}{l}\text { Data } \\
\text { mining }\end{array}$ & $\begin{array}{l}\text { Multi } \\
\text { agent } \\
\text { systems }\end{array}$ & $\begin{array}{l}\text { Service } \\
\text { oriented } \\
\text { architecture }\end{array}$ & $\begin{array}{l}\text { Data } \\
\text { warehousing }\end{array}$ & $\begin{array}{l}\text { Outcome } \\
\text { prediction }\end{array}$ & $\begin{array}{l}\text { Parent } \\
\text { decision } \\
\text { support }\end{array}$ & $\begin{array}{l}\text { Physician } \\
\text { decision } \\
\text { support }\end{array}$ & Monitoring & $\begin{array}{l}\text { Treatment } \\
\text { recommendation }\end{array}$ \\
\hline 2 & & $*$ & $*$ & & & & $*$ & $*$ & $*$ & $*$ & $*$ \\
\hline 3 & $*$ & & $*$ & & & & $*$ & $*$ & $*$ & & \\
\hline 23 & & & $*$ & & & & $*$ & $*$ & $*$ & $*$ & \\
\hline 20 & & & $*$ & & & & $*$ & $*$ & $*$ & & \\
\hline 21 & & & $*$ & $*$ & $*$ & & & & & $*$ & & \\
\hline 13 & & & & $*$ & $*$ & & & & $*$ & & \\
\hline P.A & & $*$ & $*$ & $*$ & & $*$ & $*$ & & $*$ & $*$ & \\
\hline
\end{tabular}

*Proposed architecture

\section{Conclusions}

It is important to provide high-risk neonates with the right treatment at the right time. Providing real-time specialized care decreases the mortality rate in the NICU. There are currently many weaknesses in managing highrisk neonates in the NICU environment. In order to overcome the current weaknesses, we applied a multi agent based analysis and design methodology as a new solution for NICU management to provide mechanisms for online data gathering, real time collaboration, and flexible management of NICU. To the best of our knowledge, use of multidisciplinary approaches that combine artificial intelligence and data mining techniques for monitoring, detection of high-risk neonates, and management of NICU processes is less common for neonatal intensive care; therefore, the main contribution of this study was applying a multi agent system providing a suitable platform for developing critical and complex systems like neonatal intensive care decision support systems. One of the weaknesses of this study was the need for a security solution to be integrated within the system regarding the agents' roles and communication. One of the limitations of multi agent systems is that the agent development environments are not mature enough for designing and maintaining real world systems. We are currently working on an outcome prediction module using artificial intelligence techniques for neonatal mortality risk prediction. Full implementation of the proposed architecture and its evaluation are considered as future works. Future studies in this field include applying artificial intelligence techniques for prognosis, diagnosis, monitoring, and outcome prediction of high-risk neonate.

Acknowledgments:

We acknowledge the support of the Maternal, Fetal, and Neonatal Research Center of Tehran University of Medical Sciences. This study was part of a PhD thesis.

\section{Conflict of Interest:}

There is no conflict of interest to be declared.

Authors' contributions:

All authors contributed to this project and article equally. All authors read and approved the final manuscript.

\section{References:}

1) Gilchrist J, Frize M, Ennett CM, Bariciak E. Neonatal mortality prediction using real-time medical measurements. IEEE International Workshop on; 2011: IEEE. doi: 10.1109/MeMeA.2011.5966653.

2) Frize M, Weyand S, Bariciak E, Gilchrist J, Dunn S, Tozer S. Combined Physician-Parent Decision Support tool for the neonatal intensive care unit. Medical Measurements and Applications Proceedings (MeMeA), IEEE; 2011. doi: 10.1109/MeMeA.2011.5966652.

3) Frize M, Yang L, Walker RC, O'Connor AM. Conceptual framework of knowledge management for ethical decision-making support in neonatal intensive care. IEEE Trans Inf Technol Biomed. 2005; 9(2): 205 15.doi: 10.1109/TITB.2005.847187. PMID: 16138537.

4) Frize M, Walker R. Clinical decision-support systems for intensive care units using case-based reasoning. Med Eng Phys. 2000; 22(9): 671-7. doi: 10.1016/S1350-4533(00)00078-3. PMID: 11259936.

5) Frize M, Walker RC, Ennett CM. Development of an evidence-based ethical decision-making tool for neonatal intensive care medicine. Engineering in Medicine and Biology Society, 2003 Proceedings of the 25th Annual International Conference of the IEEE; 2003. doi: 10.1109/IEMBS.2003.1279490. 
6) Santos MF, Portela F, Vilas-Boas M. INTCARE: multi-agent approach for real-time intelligent decision support in intensive medicine. 2011.

7) Malak JS, Mohsenzadeh M, Seyyedi MA. Web service qos prediction based on multi agents. Computer Technology and Development, ICCTD'09 International Conference. IEEE; 2009. doi: 10.1109/ICCTD.2009.79.

8) Foster D, McGregor C, El-Masri S. A survey of agent-based intelligent decision support systems to support clinical management and research. Proceedings of the 2nd International Workshop on Multi-Agent Systems for Medicine, Computational Biology, and Bioinformatics; 2005.

9) Julian V, Botti V. Developing real-time multi-agent systems. Integrated Computer-Aided Engineering. 2004; 11(2): 135-49.

10) Safdari R, Shoshtarian Malak J, Mohammadzadeh N, Danesh Shahraki A. A Multi Agent Based Approach for Prehospital Emergency Management. Bull Emerg Trauma. 2017; 5(3): 171-8. PMID: 28795061, PMCID: PMC5547204.

11) Temko A, Marnane W, Boylan G, Lightbody G. Clinical implementation of a neonatal seizure detection algorithm. Decis Support Syst. 2015; 70: 86-96. doi: 10.1016/j.dss.2014.12.006. PMID: 25892834, PMCID: PMC4394138.

12) Mohammadzadeh N, Safdari R, Rahimi A. Multi-agent systems: effective approach for cancer care information management. Asian Pacific Journal of Cancer Prevention. 2013; 14(12): 7757-9. doi: 10.7314/APJCP.2013.14.12.7757. PMID: 24460364.

13) Foster D, McGregor C. Design of an agent server for neonatal analysis and trend detection. The Second International Workshop on Multi-Agent Systems for Medicine, Computational Biology, and Bioinformatics; 2006.

14) Mohammadzadeh N, Safdari R. Chronic heart failure follow-up management based on agent technology. Healthc Inform Res. 2015; 21(4): 307-14. doi: 10.4258/hir.2015.21.4.307. PMID: 26618038, PMCID: PMC4659889.

15) McArthur SD, Davidson EM, Catterson VM, Dimeas AL, Hatziargyriou ND, Ponci F, et al. Multi-agent systems for power engineering applications-Part I: Concepts, approaches, and technical challenges. IEEE Transactions on Power systems. 2007; 22(4): 1743-52. doi: 10.1109/TPWRS.2007.908471.

16) Poslad S. Specifying protocols for multi-agent systems interaction. ACM Transactions on Autonomous and Adaptive Systems (TAAS). 2007; 2(4):15. doi: 10.1145/1293731.1293735.

17) Lin H. Architectural Design of Multi-Agent Systems: Technologies and Techniques: IGI Global; 2007.

18) Mrozek D, Małysiak-Mrozek B, Waligóra I. UMAP-A Universal Multi-Agent Platform for. NET Developers. International Conference: Beyond Databases, Architectures and Structures. Springer; 2014. doi: 10.1007/978-3-319-06932-6 29.

19) Mrozek D. M-MB, Waligóra I. UMAP-Universal Multi-Agent Platform for. NET developers .Available from: http://www.zti.aei.polsl.pl/w3/dmrozek/umap.htm.

20) Frize M, Gilchrist J, Martirosyan H, Bariciak E. Integration of outcome estimations with a clinical decision support system: Application in the neonatal intensive care unit (NICU). Medical Measurements and Applications (MeMeA). IEEE International Symposium. IEEE; 2015. doi: 10.1109/MeMeA.2015.7145194.

21) Guimarães T, Coimbra C, Portela F, Santos MF, Machado J, Abelha A. Step towards Multiplatform Framework for Supporting Pediatric and Neonatology Care Unit Decision Process. Procedia Computer Science. 2015; 63: 561-8. doi: 10.1016/j.procs.2015.08.385.

22) Cernuzzi L, Juan T, Sterling L, Zambonelli F. The gaia methodology. Methodologies and Software Engineering for Agent Systems. 2004; 69-88. doi: 10.1007/1-4020-8058-1_6.

23) Jemal H, Kechaou Z, Ayed MB. Towards a Medical Intensive Care Unit Decision Support System Based on Intuitionistic Fuzzy Logic. International Conference on Intelligent Systems Design and Applications. Springer; 2016.

24) Jemmaa $A B$, Ltifi $H$, Ayed $M B$. Multi-agent architecture for visual intelligent remote healthcare monitoring system. Hybrid Intelligent Systems. 2016; 211-21.

25) Ellouzi H, Ltifi H, Ben Ayed M. Multi-agent modelling of decision support systems based on visual data mining. Multiagent and Grid Systems. 2017; 13(1): 31-45. doi: 10.3233/MGS-170260.

26) Dubovitskaya A, Urovi V, Barba I, Aberer K, Schumacher MI. A Multiagent System for Dynamic Data Aggregation in Medical Research. Bio Med Research International. 2016; 2016. doi: $10.1155 / 2016 / 9027457$. 